\title{
Sleep enhances category learning
}

\author{
Ina Djonlagic, ${ }^{1,2,6}$ Andrew Rosenfeld, ${ }^{1}$ Daphna Shohamy, ${ }^{3}$ Catherine Myers, ${ }^{4}$ \\ Mark Gluck, ${ }^{5}$ and Robert Stickgold ${ }^{1}$ \\ ${ }^{1}$ Center for Sleep and Cognition, Beth Israel Deaconess Medical Center, Boston, Massachusetts 02215, USA; ${ }^{2}$ Division of Sleep \\ Medicine, Brigham and Women's Hospital, Harvard Medical School, Boston, Massachusetts 02115, USA; ${ }^{3}$ Psychology \\ Department, Columbia University, New York, New York 10027, USA; ${ }^{4}$ Department of Psychology, Rutgers University, Newark, \\ New Jersey 07102, USA; ${ }^{5}$ Center for Molecular and Behavioral Neuroscience, Rutgers University, Newark, New Jersey 07102, USA
}

\begin{abstract}
The ability to categorize objects and events in the world around us is a fundamental and critical aspect of human learning. We trained healthy adults on a probabilistic category-learning task in two different training modes. The aim of this study was to see whether either form of probabilistic category learning (feedback or observational) undergoes subsequent enhancement during sleep. Our results suggest that after training, a good night of sleep can lead to improved performance the following day on such tasks.
\end{abstract}

[Supplemental material is available online at http://www.learnmem.org.]

From infancy, humans categorize objects and events they encounter. In our daily lives, this category learning enables us to extract rules and patterns from our varied experiences, and to formulate new responses and inferences to familiar objects and events, thereby facilitating decision-making and problem-solving processes (Gluck et al. 2008).

While there is now considerable understanding of the early stages of category learning, relatively little is known about how such learning evolves over time. A growing literature shows that post-training sleep plays an important role in long-term memory consolidation and enhancement (Stickgold 2005; Diekelmann et al. 2009). The most compelling evidence thus far comes from simple perceptual and motor procedural learning, such as fingertapping sequences and visual discrimination tasks (Karni et al. 1994; Gais et al. 2000; Stickgold et al. 2000; Walker et al. 2002; Huber et al. 2004). Much less is understood about the role of sleep in the learning of explicit episodic memory tasks, and even less for more complex memory tasks, such as probabilistic category learning (Smith and Smith 2003; Wagner et al. 2004; Ellenbogen et al. 2007).

There are two basic patterns by which categorizations can be learned: We can simply observe stimuli and their outcomes (observational learning), or we can be shown stimuli and then asked to predict outcomes, only then receiving feedback on the accuracy of our response (feedback learning). The Weather Prediction Task (WPT) was initially described by Knowlton et al. (1994) and has been extensively studied as a paradigm for such category learning (Knowlton et al. 1994; Gluck et al. 2002; Meeter et al. 2008).

Studies have shown that the WPT activates different memory systems depending on how the task is learned and on the extent of training. Neuroimaging studies have shown that subjects who simply observe stimuli and their outcomes activate learning and memory systems that are supported by the medial temporal lobe (MTL) and prefrontal cortex (observational learning, Fig. 1, top). On the other hand, subjects who must predict outcomes for each stimulus and only then receive feedback on their accuracy (feedback learning, Fig. 1, bottom), initially activate the MTL,

\footnotetext{
${ }^{6}$ Corresponding author.
}

E-mail idjonlag@bidmc.harvard.edu; fax (617) 667-8498.

Article is online at http://www.learnmem.org/cgi/doi/10.1101//m.1634509. but as training continues shift this activation to the striatum, which is known to be involved with habit and skill-learning behaviors (Poldrack et al. 2001).

Evidence from studies with patient populations supports this conclusion. Patients in whom basal ganglia are affected, such as those with Parkinson disease, Huntington disease, and Tourette syndrome, are impaired in feedback-based probabilistic category learning, whereas patients with amnesia following MTL or diencephalic damage show intact feedback learning early, but not later in training (Knowlton et al. 1996a,b; Keri et al. 2005). But, when the same task was learned in observational mode, Parkinson patients showed no learning impairment, suggesting activation of brain areas that are spared in Parkinson disease, such as the MTL (Shohamy et al. 2004a).

Whether either form of learning undergoes subsequent sleepdependent enhancement remains unknown. Thus, the aim of this study was to see whether either form of probabilistic category learning (feedback or observational) undergoes subsequent enhancement during sleep.

Healthy college students $18-25$ yr of age were recruited and randomly assigned to undergo training on the WPT in either observational or feedback mode after they provided IRB-approved informed consent. To ensure sleep-wake homogeneity, subjects were required to sleep, on average, $7 \mathrm{~h}$ per night and to maintain their normal sleep schedule for $2 \mathrm{~d}$ prior to the experiment as well as between test sessions if it included a night of sleep. In addition, participants abstained from caffeine and alcohol throughout the experiment and had to refrain from napping on protocol days. Subjects were excluded if they (1) had a history of alcohol, narcotic, or other drug abuse; (2) had a history of psychiatric or neurologic disorders; (3) had a diagnosed sleep disorder; (4) used medications known to have an effect on sleep and daytime vigilance (e.g., psychoactive drugs or medications, sedatives, or hypnotics); or (5) consumed over $600 \mathrm{mg}$ per day of caffeine.

The WPT consists of four cards with geometric patterns, which serve as stimulus elements. On each trial, subjects see one, two, or three of these cards in varying combinations, and use this aggregate stimulus to predict one of two possible weather outcome categories: sunshine or rain. The weather outcome is determined by a probabilistic rule, with each card having a fixed probability of predicting sunshine and rain, which each occur equally often. The final outcome is based on a combined probability 


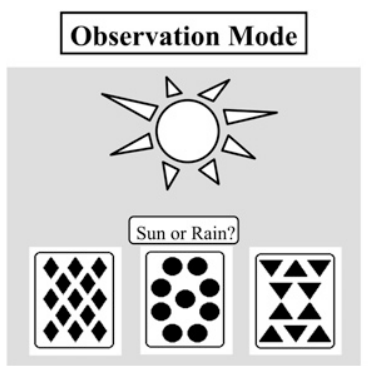

Feedback Mode

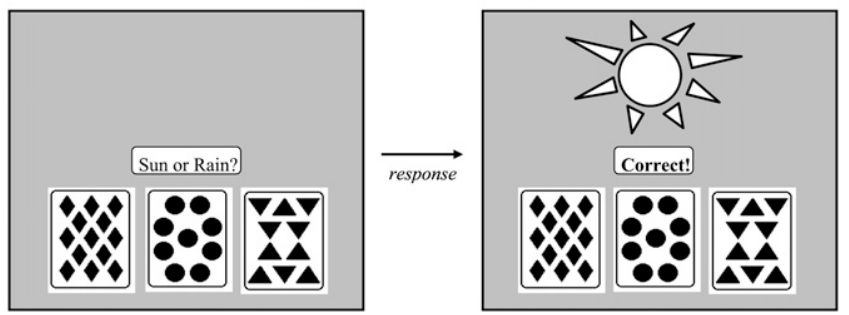

Figure 1. Sample screens of WPT stimulus and response screens. (Top) Observation mode. On each trial, subjects see a set of cards along with the correct weather outcome displayed above. They then press the corresponding key ("sun" or "clouds") to move to the next trial. (Bottom) Feedback mode. On each trial, subjects see a set of cards and are asked to predict the correct weather outcome by pressing either the "sun" or "clouds" key. After pressing a key the actual outcome is displayed above the cards, along with the information on whether their prediction had been correct or incorrect.

associated with the overall stimulus. Because of the probabilistic nature of the task, information from a single trial is not a reliable indicator of how well the task was learned. Instead, information accrued over many trials is used for analysis.

During the task, subjects are seated in front of a computer and are instructed to pay close attention during the following trials since they will later be asked to remember what kind of weather the cards predict. They are not given explicit information about the probabilistic nature of the stimulus-outcome relationship. Training involves 200 trials consisting of 14 patterns of card combinations with a fixed order for all subjects. The keyboard keys for "sun" and "clouds" are marked. (For a more detailed description of observational and feedback-based training, please see Supplemental material.) After the training phase in either observational or feedback mode, instructions appear on the screen indicating that a new phase is beginning. At the beginning of the test phase, subjects are told that the same cards and combinations will be displayed on the screen and that their job is to predict the weather by pressing either the "sun" or the "clouds" key. After the subject responds to each card combination, the program moves to the next trial without giving feedback. Testing consists of 100 trials, also with a fixed order for all subjects. When subjects return $12 \mathrm{~h}$ later, they undergo a second test phase. This test phase consists of the same 100 trials with the identical trial sequence as in the first test session. Again, no feedback is given.

Performance on the WPT was scored as the percent of optimal responses achieved during a test session. An optimal response is the response that is more frequently associated with a given card pattern. Raw performance on the WPT was subsequently converted to "adjusted performance," which adjusts for the $50 \%$ of correct responses that are expected by chance, and represent the best estimate of the actual percent of trials correctly answered because of training. (Adjusted score $=$ [raw score -0.5$] / 0.5$.) Improvement from Test 1 to Test 2, then, is adjusted score [2] - adjusted score [1], which is mathematically equivalent to $2 \times$ (raw score [2] - raw score [1]).

Subjects assigned to observational training were then further divided into a Wake and a Sleep group. The Wake group $(n=20)$ received 200 training trials at $9 \mathrm{am}$, immediately followed by 100 test trials with no feedback, and then were retested that evening at $9 \mathrm{pm}$; while subjects in the Sleep group $(n=15)$ were initially trained and tested at $9 \mathrm{pm}$, before being retested after a night of normal sleep, at 9 am the following morning.

No significant performance differences were found between the Wake and Sleep groups in the immediate post-training test session (Wake 77.0 $\pm 2.9 \%$ [SEM], Sleep $=74.8 \pm 3.4 \% ; P=0.61$ ). But $12 \mathrm{~h}$ later, the Sleep group showed significantly more improvement than the Wake group $(13.0 \pm 1.9 \%$ vs. $3.5 \pm$ $1.3 \%, P=0.0002$; Fig. $2 \mathrm{~A}$ ).

To investigate the relationship between overnight improvement and sleep parameters, a second Sleep group $(n=16)$ was trained in the observation mode and had their sleep recorded with overnight polysomnography between test sessions. They were trained on the WPT at $9 \mathrm{pm}$ in observational mode. After the immediate testing session, they were wired for standard overnight polysomnographic (PSG) recording in the Harvard-Thorndike General Clinical Research Center (GCRC) at Beth Israel Deaconess
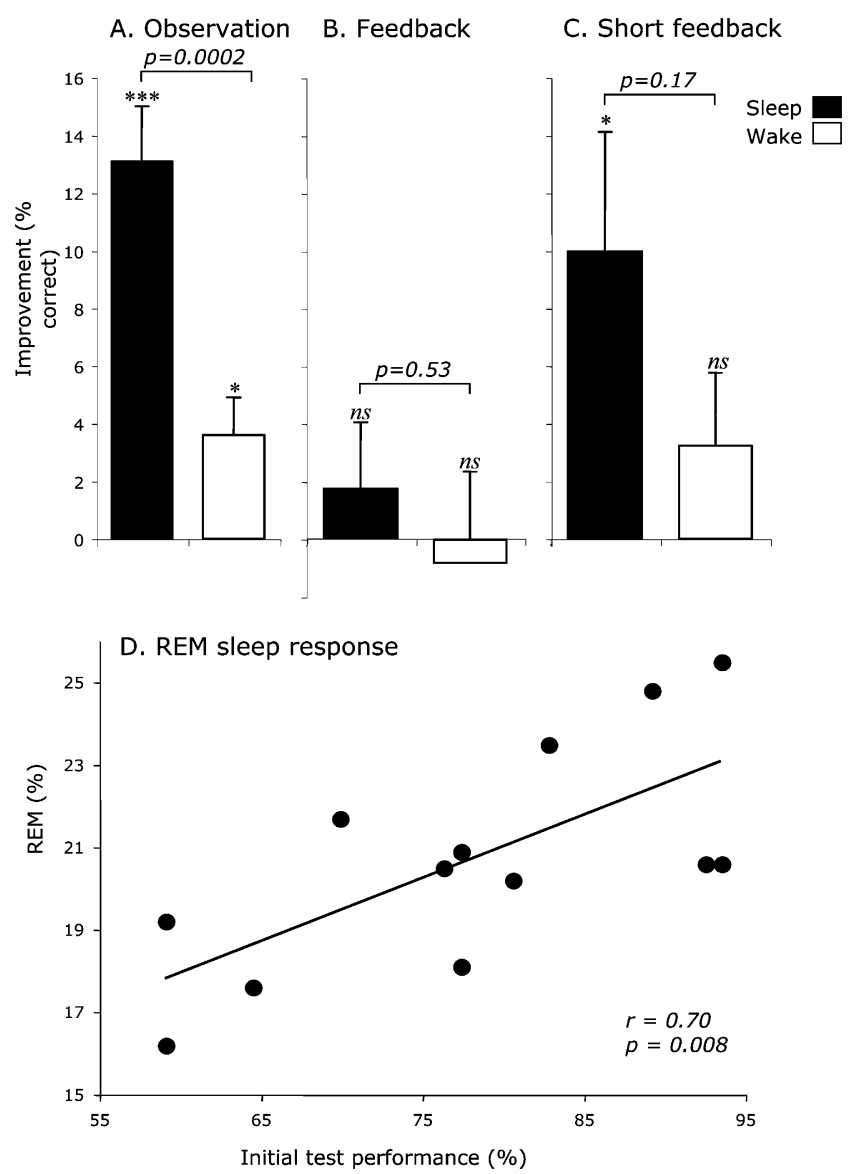

Figure 2. Sleep's effect on WPT performance. (A-C) Corrected improvement \pm SEM is shown. Significant improvement was seen in the Sleep groups trained with either 200 trials in observational mode $(A)$ or with 100 trials in short feedback mode (C), but not with 200 trials in feedback mode $(B)$. Subjects in the Wake groups failed to show significant improvement in feedback mode $(B, C)$. $(D)$ Subjects who performed well at the initial test session had a higher percentage of REM sleep the following night. $\left(^{*}\right) P<0.05 ;\left(^{* * *}\right) P<0.0001$. 
Medical Center. The standard PSG montage consisted of a fourchannel electroencephalogram (EEG), recording channels C4/A1, $\mathrm{C} 3 / \mathrm{A} 2$, O1/A2, and O2/A1. In addition, the montage included a bilateral electrooculogram (EOG), and a submental electromyogram (EMG). All PSG data were recorded with the MedCare Embla Somnologica digital recording system. When subjects awoke the next morning (after no more than $9 \mathrm{~h}$ in bed), electrodes were removed prior to retesting at $9 \mathrm{am}$. Sleep stages were scored according to the criteria of Rechtschaffen and Kales (1968).

This second observational Sleep group showed a strong correlation between their initial learning performance and the amount of REM sleep they obtained the following night $(r=0.70$, $P=0.008$; Fig. 2D). Unexpectedly, however, overnight improvement was not significant $(3.2 \pm 2.0 \%, P=0.14)$, perhaps due to the unfamiliar conditions of the sleep laboratory.

This correlation between REM sleep and performance is consistent with past animal and human studies showing that an increased amount of post-training REM sleep occurs when a sufficient level of learning has occurred during prior training (Guerrien et al. 1989; Hennevin et al. 1995; Smith 1995). Studies in rats have demonstrated specific reactivation patterns of hippocampal neuronal activity during REM sleep in the same temporal sequence as they had during prior wakefulness, suggesting that circuits may be restructured during REM sleep by selectively strengthening some recently acquired memories (Guerrien et al. 1989; Hennevin et al. 1995; Smith 1995; Poe et al. 2000; Louie and Wilson 2001).

Human studies using positron emission tomography have demonstrated that brain areas that were activated when learning a probabilistic serial reaction time task during the day were significantly more active during subsequent REM sleep. In addition, reactivation of the cuneus during REM sleep was dependent on and modulated by the level of prior learning on the probabilistic version of the task, but not on the level of general acquisition of basic visuomotor skills seen with a random sequence version of the task (Maquet et al. 2000; Peigneux et al. 2003). While these findings in humans suggest that successful learning leads to a reactivation similar to that seen in rodents, it remains possible that the differences in activation seen during REM sleep reflect individual trait-like differences and could indicate an individual's general learning ability (Buckelmuller et al. 2006). Previous reports about a correlation between baseline amounts of REM sleep and learning potentials, however, have been inconsistent. Some studies have shown a positive association with REM sleep and intelligence (Petre-Quadens and De Lee 1970; Pagel et al. 1973); others have shown the opposite (Busby and Pivik 1983), and Smith et al. (2004) proposed a model suggesting that the posttraining REM response following task acquisition is partly genetically determined and partly a response to the task itself.

To determine whether learning the same WPT rules (but using feedback-based learning) also showed sleep-dependent enhancement, we trained two groups of subjects on the WPT in feedback mode and then retested them after either $12 \mathrm{~h}$ of wake $(n=11)$ or $12 \mathrm{~h}$ including a night of sleep $(n=10)$. In feedback training, subjects were shown each pattern and then had to predict its associated weather category before being shown the outcome. While the training mode differed, subjects were presented with the same 200 stimuli and outcomes in training, and the same 100 test trials as subjects in the observational mode.

As in the observational mode, Wake and Sleep subjects showed similar performances in the immediate post-training test session $($ Wake $=81.5 \pm 4.3 \%$, Sleep $=90.5 \pm 1.1, P=0.07)$. In contrast to the observational training, there was no significant improvement in either feedback group at retest (Wake $=-0.8 \pm$ $3.1 \%, P=0.81$; Sleep $=1.7 \pm 2.3 \%, P=0.47)$ and no significant difference between the groups $(P=0.53$; Fig. $2 \mathrm{~B})$. Interpretation of these results must be tempered, however, by possible ceiling effects in the Sleep group, which had exceptionally high initial performance $(90.5 \%)$.

Taken as a group, subjects trained in the feedback mode demonstrated higher initial performance scores than those trained in the observational mode (feedback $=85.8 \pm 2.5 \%$, observational $=$ $76.1 \pm 2.2 \%, P=0.006$ ). This difference cannot be explained by more optimal responses during the 200-trial training session in the feedback mode for either the Wake groups (observational $=82.6 \%$, feedback $=80.9 \% ; P=0.226$ ) or the Sleep groups (observational $=$ $81.8 \%$, feedback $=77.4 \% ; P=0.153$ ). Instead, feedback-based learning appears to lead to higher performance scores during subsequent testing through activation of different neural systems than those used in observational training.

To test whether the sleep-dependent improvement seen in the observational group was dependent on the lower level of initial training performance or on the training mode by which it was learned, we trained a third pair of Wake and Sleep groups using an abbreviated version of the feedback mode ("short feedback mode") with only 100 training trials, and retested them after periods of wake or sleep.

Wake and Sleep groups again demonstrated similar initial posttraining performance levels $($ Wake $=74.0 \pm 4.5 \%$, Sleep $=74.4 \pm$ $2.7 \%, P=0.94)$ and levels similar to the observational groups (short feedback $=74.2 \pm 2.6 \%$, observational $=76.1 \pm 2.2 \%, P=$ $0.59)$, although at levels lower than the standard feedback groups.

The Sleep group $(n=13)$ now showed significant improvement $(9.9 \pm 4.1 \%, P=0.032)$, while the Wake group $(n=14)$ still did not ( $3.2 \pm 2.5 \%, P=0.22$; Fig. $2 \mathrm{C})$. Improvement in the short feedback mode did not differ significantly from that in the observational mode for either the Sleep groups $(9.9 \pm 4.1 \%$ vs. $13.0 \pm 1.9 \%, P=0.48)$ or the Wake groups $(3.2 \pm 2.5 \%$ vs. $3.5 \pm$ $1.3 \%, P=0.90$; Fig. 2, cf. A and C). These findings suggest that the extent of overnight improvement is dependent on the level of performance achieved during training. Indeed, significant correlations between post-training scores and improvement were present in the Sleep groups, but not in the Wake groups. In the Sleep groups, maximal improvement over time was seen in those subjects with relatively poor post-training performance. While this correlation was highly significant for the Sleep groups $(r=-0.57$, $P<0.0001$; Fig. 3B, heavy dashed line), no correlation was seen for the Wake groups $(r=-0.037, P=0.81$; Fig 3A, heavy dashed line). Individual Sleep groups showed similar correlations (see Supplemental material). Although the maximum possible improvement decreases as initial post-training performance approaches $100 \%$, scores fell decidedly below these limits (Fig. 3, light dashed lines), suggesting that the correlation is not a simple consequence of ceiling effects. A similar pattern, showing greater sleep-dependent benefits with poorer initial performance, has been reported for a finger-tapping motor sequence task (Kuriyama et al. 2004).

Immediately after subjects finished the second test session, they completed a questionnaire assessing their explicit knowledge of the relationships between the four cards and the weather outcome. Subjects were first asked which card(s) most often predicted "sun" and "rain." Then, for each of the four cards, they indicated what percent of the time they believed each card was followed by sunshine and by clouds. Ratings were subsequently compared with two theoretical models of how subjects would explicitly understand the task. In one model, subjects would assign each individual card with probabilities close to those actually observed (24\%-76\% sunshine). But, in the second model, subjects would dichotomize the four cards into two cards that always predicted sunshine and two that always predicted rain.

For the combined observational and short feedback conditions, each of which showed sleep-dependent improvement in performance, subjects in the Wake groups were more than three 


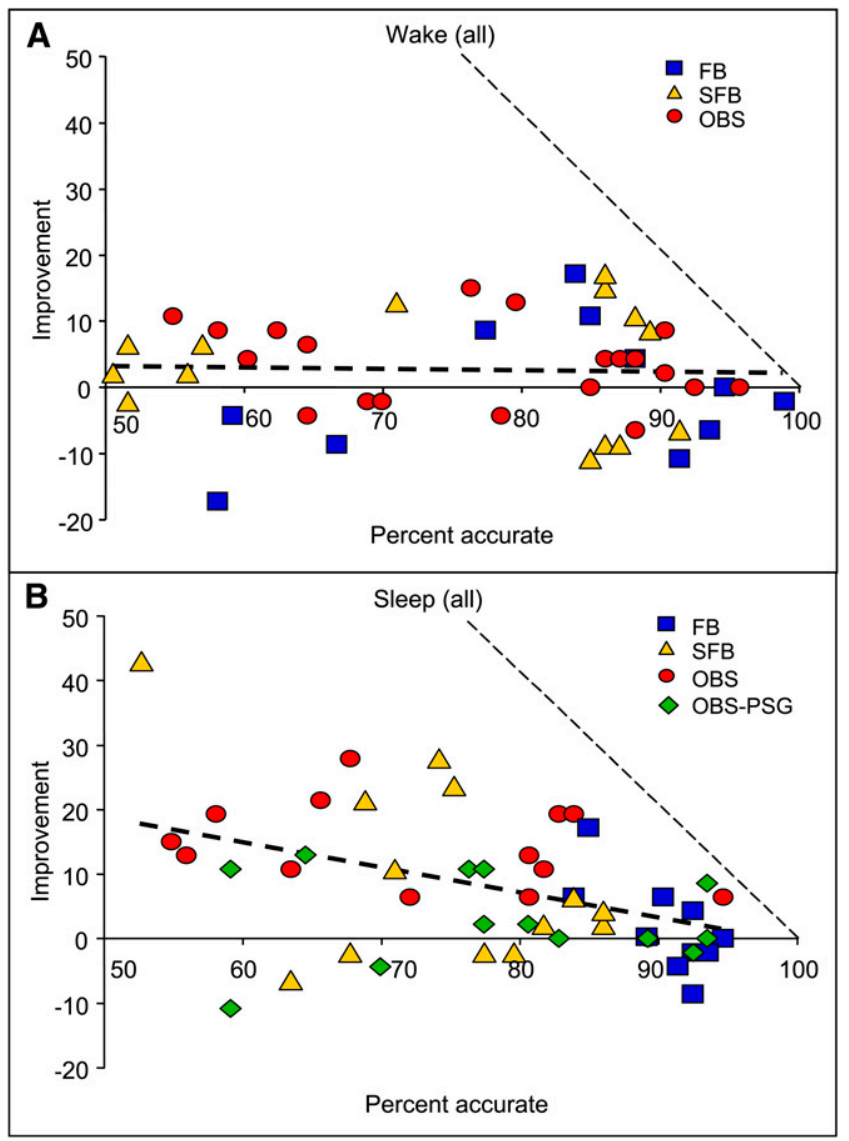

Figure 3. Correlation between post-training performance and $12-\mathrm{h}$ improvement. (OBS) Observational mode; (FB) feedback; (SFB) short feedback; (PSG) observational mode in sleep laboratory. Light dashed lines: ceiling limits; heavy dashed lines: regression fits to all points. Specifics regarding panels $A$ and $B$ in text.

times as likely to estimate probabilities of $0 \%$ and $100 \%$ (dichotomized) for the circle and diamond cards, which had actual rates of $42 \%$ and $58 \%$ sunshine $\left(P=0.02, \chi^{2}=5.4, d f=1\right)$. In addition, individual differences in ratings of circles and diamonds did not differ significantly from the actual difference for the Sleep group $\left(22.9 \% \pm 8.5\right.$; compared with $\mathrm{h}_{0}=16, d f=25, t=0.81, P=$ $0.43)$, while the Wake group showed a significantly inflated difference $(56.9 \pm 8.7, d f=30, t=4.72, P<0.001)$, and the difference between the Wake and Sleep group was significant $(P=$ $0.007, d f=55, t=2.78$ ). Individually, the observational and short feedback conditions show the same patterns (for complete distribution of estimations for all four cards see Supplemental material). Both feedback groups, Wake and Sleep, dichotomize the cards and there is no difference between them.

The same bias toward dichotomizing in the Wake group was seen implicitly during the final test session. Subjects in the Wake groups (short feedback and observational) were more likely to dichotomize their performance on trials with the circles or diamonds the evening after training than subjects in the Sleep group, who were tested following a night of sleep $(P=0.008$, $\left.\chi^{2}=7.41, d f=1\right)$. Wake subjects again showed higher mean differences in scores for circles and diamonds (percent "sunshine" responses for circles - diamonds $=74.2 \% \pm 9.0$ ) than the Sleep group $(41.3 \% \pm 12.0$; Wake vs. Sleep: $d f=55, t=2.24, P=0.03)$ (see also additional figures in the Supplemental material).

The findings reported here represent the first clear evidence that active, off-line memory enhancement of a procedural cate- gory rule-learning task takes place throughout a night of sleep, leading to an absolute improvement in performance the next morning. The strongest evidence was seen when comparing Sleep and Wake conditions in the observational group $(P=0.0002)$. While the direct sleep-wake comparison was not significant in the short feedback condition, there was a significant $10 \%$ improvement after sleep $(P=0.03)$, while the matched Wake group showed only a nonsignificant $3 \%$ improvement $(P=0.22)$. In addition, there was a trend toward a correlation between initial performance and subsequent improvement in the Sleep group $(r=-0.50, P=$ $0.08)$, but no hint of a correlation in the Wake group $(r=0.05, P=$ 0.85), further supporting the possibility that sleep leads to improved performance on the WPT in short feedback condition.

Some evidence, mainly from animal studies, has suggested a circadian influence on synaptic plasticity, particularly in the hippocampus (Craig and McDonald 2008; Ruby et al. 2008). Thus, as with any study comparing memory consolidation over a period of wakefulness vs. sleep, we cannot exclude the possibility of additional circadian effects on our results. However, the fact that performance on the WPT shows an absolute improvement across a night of sleep, as opposed to simply showing less deterioration than seen across periods of wake, rules out the possibility that sleep only offers passive protection of newly encoded memory traces from daytime interference. Rather, sleep must support active memory consolidation.

The positive correlation between the extent of learning (measured by performance following the completion of training) and the amount of REM sleep obtained the following night suggests that successful learning of the classification of objects and events in the world around us can lead to an increase in subsequent REM sleep, and provides further evidence of an active, sleep-dependent process.

The brain mechanisms that produce these sleep-dependent improvements of memory remain elusive. Yet, the multifaceted relationship between the type and quantity of training, on the one hand, and the amount of overnight improvement, on the other, introduces a new level of complexity to our understanding of how sleep enhances memory. The fact that significant sleep-dependent improvement on the WPT was seen only for subjects who demonstrated an intermediate level of learning during trainingirrespective of how the task was acquired-may provide some hints. Evidence from previous imaging studies and studies using patients with amnesia and Parkinson's disease suggests that memory systems supported by the MTL and prefrontal cortex are activated during observational learning and early stages of feedback learning (using 100 trials), while the striatum is activated as feedback training continues (Poldrack et al. 2001; Aron et al. 2004; Hopkins et al. 2004; Shohamy et al. 2004a,b).

Thus, one intriguing mechanism that could explain our findings is that a localized sleep-dependent process strengthens memories stored in hippocampal-neocortical networks, but not those stored in the striatum (Marshall and Born 2007).

\section{Acknowledgments}

This work was supported by NIH grant MH48832 and National Center for Research Resources grants M01RR001032 and NIH-T32HL07901-06.

\section{References}

Aron AR, Shohamy D, Clark J, Myers C, Gluck MA, Poldrack RA. 2004. Human midbrain sensitivity to cognitive feedback and uncertainty during classification learning. J Neurophysiol 92: 1144-1152.

Buckelmuller J, Landolt HP, Stassen HH, Achermann P. 2006. Trait-like individual differences in the human sleep electroencephalogram. Neuroscience 138: 351-356. 
Busby K, Pivik RT. 1983. Sleep patterns in children of superior intelligence. $J$ Child Psychol Psychiatry 24: 587-600.

Craig LA, McDonald RJ. 2008. Chronic disruption of circadian rhythms impairs hippocampal memory in the rat. Brain Res Bull 76: 141151.

Diekelmann S, Wilhelm I, Born J. 2009. The whats and whens of sleepdependent memory consolidation. Sleep Med Rev 13: 309-321.

Ellenbogen JM, Hu PT, Payne JD, Titone D, Walker MP. 2007. Human relational memory requires time and sleep. Proc Natl Acad Sci 104: 7723-7728.

Gais S, Plihal W, Wagner U, Born J. 2000. Early sleep triggers memory for early visual discrimination skills. Nat Neurosci 3: 1335-1339.

Gluck MA, Shohamy D, Myers C. 2002. How do people solve the "weather prediction" task?: Individual variability in strategies for probabilistic category learning. Learn Mem 9: 408-418.

Gluck MA, Poldrack RA, Keri S. 2008. The cognitive neuroscience of category learning. Neurosci Biobehav Rev 32: 193-196.

Guerrien A, Dujardin K, Mandai O, Sockeel P, Leconte P. 1989. Enhancement of memory by auditory stimulation during postlearning REM sleep in humans. Physiol Behav 45: 947-950.

Hennevin E, Hars B, Maho C, Bloch V. 1995. Processing of learned information in paradoxical sleep: Relevance for memory. Behav Brain Res 69: 125-135.

Hopkins RO, Myers CE, Shohamy D, Grossman S, Gluck M. 2004. Impaired probabilistic category learning in hypoxic subjects with hippocampal damage. Neuropsychologia 42: 524-535.

Huber R, Ghilardi MF, Massimini M, Tononi G. 2004. Local sleep and learning. Nature 430: $78-81$.

Karni A, Tanne D, Rubenstein BS, Askenasy JJ, Sagi D. 1994. Dependence on REM sleep of overnight improvement of a perceptual skill. Science 265: 679-682.

Keri S, Nagy O, Kelemen O, Myers CE, Gluck MA. 2005. Dissociation between medial temporal lobe and basal ganglia memory systems in schizophrenia. Schizophr Res 77: 321-328.

Knowlton BJ, Squire LR, Gluck MA. 1994. Probabilistic classification learning in amnesia. Learn Mem 1: 106-120.

Knowlton BJ, Squire LR, Paulsen JS, Swerdlow NR, Swenson M. 1996a. Dissociations within nondeclarative memory in Huntington's disease. Neuropsychology 10: 538-548.

Knowlton BJ, Mangels JA, Squire LR. 1996b. A neostriatal habit learning system in humans. Science 273: 1399-1402.

Kuriyama K, Stickgold R, Walker MP. 2004. Sleep-dependent learning and motor-skill complexity. Learn Mem 11: 705-713.

Louie K, Wilson MA. 2001. Temporally structured replay of awake hippocampal ensemble activity during rapid eye movement sleep. Neuron 29: 145-156.

Maquet P, Laureys S, Peigneux P, Fuchs S, Petiau C, Phillips C, Aerts J, Del Fiore G, Degueldre C, Meulemans T, et al. 2000. Experience-dependent changes in cerebral activation during human REM sleep. Nat Neurosci 3: 831-836.

Marshall L, Born J. 2007. The contribution of sleep to hippocampusdependent memory consolidation. Trends Cogn Sci 11: 442-450.
Meeter M, Radics G, Myers CE, Gluck MA, Hopkins RO. 2008. Probabilistic categorization: How do normal participants and amnesic patients do it? Neurosci Biobehav Rev 32: 237-248.

Pagel J, Pegram V, Vaughn S, Donaldson P, Bridgers W. 1973. The relationship of REM sleep with learning and memory in mice. Behav Biol 9: 383-388.

Peigneux P, Laureys S, Fuchs S, Destrebecqz A, Collette F, Delbeuck X, Phillips C, Aerts J, Del Fiore G, Degueldre C, et al. 2003. Learned material content and acquisition level modulate cerebral reactivation during posttraining rapid-eye-movements sleep. Neuroimage 20: 125134.

Petre-Quadens O, De Lee C. 1970. Eye-movements during sleep: A common criterion of learning capacities and endocrine activity. Dev Med Child Neurol 12: 730-740.

Poe GR, Nitz DA, McNaughton BL, Barnes CA. 2000. Experience-dependent phase-reversal of hippocampal neuron firing during REM sleep. Brain Res 855: 176-180.

Poldrack RA, Clark J, Pare-Blagoev EJ, Shohamy D, Creso Moyano J, Myers C, Gluck MA. 2001. Interactive memory systems in the human brain. Nature 414: 546-550.

Rechtschaffen A, Kales A. 1968. A manual standardized terminology, techniques and scoring system for sleep stages of human subjects. U.S. Department of Health, Bethesda, MD.

Ruby NF, Hwang CE, Wessells C, Fernandez F, Zhang P, Sapolsky R, Heller HC. 2008. Hippocampal-dependent learning requires a functional circadian system. Proc Natl Acad Sci 105: 15593-15598.

Shohamy D, Myers CE, Grossman S, Sage J, Gluck MA, Poldrack RA. 2004a. Cortico-striatal contributions to feedback-based learning: Converging data from neuroimaging and neuropsychology. Brain 127: 851-859.

Shohamy D, Myers CE, Onlaor S, Gluck MA. 2004b. Role of the basal ganglia in category learning: How do patients with Parkinson's disease learn? Behav Neurosci 118: 676-686.

Smith C. 1995. Sleep states and memory processes. Behav Brain Res 69: 137 145.

Smith C, Smith D. 2003. Ingestion of ethanol just prior to sleep onset impairs memory for procedural but not declarative tasks. Sleep 26: 185191.

Smith CT, Nixon MR, Nader RS. 2004. Posttraining increases in REM sleep intensity implicate REM sleep in memory processing and provide a biological marker of learning potential. Learn Mem 11: 714-719.

Stickgold R. 2005. Sleep-dependent memory consolidation. Nature 437: $1272-1278$.

Stickgold R, James L, Hobson JA. 2000. Visual discrimination learning requires sleep after training. Nat Neurosci 3: 1237-1238.

Wagner U, Gais S, Haider H, Verleger R, Born J. 2004. Sleep inspires insight. Nature 427: 352-355.

Walker MP, Brakefield T, Morgan A, Hobson JA, Stickgold R. 2002. Practice with sleep makes perfect: Sleep-dependent motor skill learning. Neuron 35: 205-211.

Received September 16, 2009; accepted in revised form September 21, 2009. 


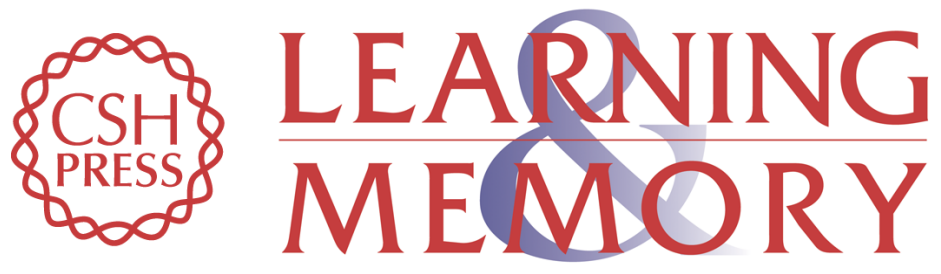

\section{Sleep enhances category learning}

Ina Djonlagic, Andrew Rosenfeld, Daphna Shohamy, et al.

Learn. Mem. 2009, 16:

Access the most recent version at doi:10.1101//m.1634509

Supplemental http://learnmem.cshlp.org/content/suppl/2009/11/30/16.12.751.DC1

Material

References This article cites 38 articles, 8 of which can be accessed free at:

http://learnmem.cshlp.org/content/16/12/751.full.html\#ref-list-1

License

Email Alerting Receive free email alerts when new articles cite this article - sign up in the box at the Service top right corner of the article or click here. 